\title{
Serum and follicular fluid concentration of stem cell factor in PCOS
}

\section{Ziya Kalem¹, Müberra Namli Kalem ${ }^{2 *}$, Murat Seval ${ }^{3}$, Batuhan Bakirarar ${ }^{4}$, Coskun Simsir², Canan Yılmaz ${ }^{5}$, Cem Atabekoglu' ${ }^{3}$, Timur Gurgan ${ }^{1}$}

\author{
${ }^{1}$ Department of IVF, Gurgan Clinic IVF and Women Health Center, Ankara, Turkey \\ ${ }^{2}$ Department of Obstetrics and Gynecology, Liv Hospital Ankara, Ankara, Turkey \\ ${ }^{3}$ Department of Obstetrics and Gynecology, ${ }^{4}$ Department of Biostatistic, Ankara University, Turkey \\ ${ }^{5}$ Department of Biochemistry, Gazi University, Ankara, Turkey
}

Received: 21 June 2018

Accepted: 24 July 2018

\section{*Correspondence:}

Dr. Müberra Namli Kalem,

E-mail: muberranamli@hotmail.com

Copyright: (c) the author(s), publisher and licensee Medip Academy. This is an open-access article distributed under the terms of the Creative Commons Attribution Non-Commercial License, which permits unrestricted non-commercial use, distribution, and reproduction in any medium, provided the original work is properly cited.

\begin{abstract}
Background: Polycystic ovarian syndrome (PCOS) is the most common endocrine disorder in reproductive-aged women, which affects 5 to $20 \%$ of women in the reproductive age worldwide. This study aimed to compare the levels of SCF in serum and follicular fluid of PCOS patients with those of non-PCOS group and to investigate the relationship of SCF levels with ICSI success.

Methods: This is an observational case control study that included the patients who underwent ICSI in the InfertilityIVF center at Ankara University Faculty of Medicine and in a private IVF center between March 2016 and February 2017. The study group consisted of 57 PCOS patients diagnosed according to the Rotterdam criteria and the control group consisted of 75 patients with normofollicular and regular menstrual cycles. Serum and follicular fluid samples were taken on day of oocyte retrieval. Serum and follicular fluid SCF levels were determined by ELISA using the SCF ELISA kit..

Results: Serum and follicular fluid SCF levels in PCOS patients were found to be lower than in non-PCOS group. sSCF and ffSCF were statistically significantly higher who had clinical pregnancy than those who had no clinical pregnancy in the PCOS group.

Conclusions: SCF levels are low in serum and follicular fluid in patients with PCOS and that the increase in SCF levels is associated with an increase in oocyte maturation and clinical pregnancy rates in PCOS.
\end{abstract}

Keywords: Clinical pregnancy rate, Polycystic ovary syndrome, Stem cell factor

\section{INTRODUCTION}

Polycystic ovarian syndrome (PCOS) is the most common endocrine disorder in reproductive-aged women, which affects 5 to $20 \%$ of women in the reproductive age worldwide. ${ }^{1}$ Diagnosis of PCOS is based on the presence of at least two of the following three criteria: oligomenorrhea or amenorrhea, clinical or biochemical evidence of hyperandrogenism, and polycystic ovaries on morphology. ${ }^{2}$ This heterogeneous metabolic dysfunction picture, which has not been fully elucidated yet, is also the main cause of anovulatory infertility. ${ }^{3}$ Assisted reproductive techniques (ARTs) are one of the recommended treatment modalities for infertility in PCOS. ${ }^{4}$ However, problems such as oocyte immaturity, low oocyte quality, low fertilization rate and low implantation rate decrease the success rate in the ART cycles of PCOS patients, and oocyte maturation disorder is the main determinant in reduced fertility. ${ }^{5,6}$

Stem cell factor (SCF, also called Steel factor or Kit ligand), a granulosa-derived growth factor, it exerts its affect by binding the c-Kit receptor at the oocyte surface, 
appears to be important for the regulation of early follicular development and enhancement of the production of oocyte factors, which in turn stimulates the proliferation and differentiation of the surrounding granulosa cells. ${ }^{7-9}$ AMH has a modulatory role as an ovarian/follicular autocrine/paracrine factor controlling SCF expression via the cAMP/PKA pathway. ${ }^{10}$ It was shown that high AMH levels decreases stem cell factor production from granulosa cells. ${ }^{11} \mathrm{SCF}$ has an antiapoptotic effect on oocytes. ${ }^{12}$ Tan et al demonstrated that reduced expression of SCF in serum and follicle from patients with polycystic ovary syndrome, and increased SCF level in follicular fluid was positively correlated with oocyte maturation, fertilization, and embryo quality in humans. ${ }^{13,14}$

In the present study, we aimed to compare the levels of SCF in serum and follicular fluid of PCOS patients with those of non-PCOS group and to investigate the relationship of SCF levels with ICSI success.

\section{METHODS}

This is an observational case control study that included the patients who underwent ICSI in the Infertility-IVF center at Ankara University Faculty of Medicine and in a private IVF center between March 2016 and February 2017. The study group consisted of 57 PCOS patients diagnosed according to the Rotterdam criteria, while the control group consisted of 75 patients with normofollicular and regular menstrual cycles. ${ }^{2}$ None of the participants in the present study had another major health problem. Patients with ovulation induction in the past three months, those with premature ovarian failure or ovarian dysfunction, those with endometriosis, hyperprolactinemia or thyroid disorder, those with previous ovary surgery, radiotherapy or chemotherapy were excluded from the study.

Gonadotropin-releasing hormone (GnRH) agonist and $\mathrm{GnRH}$ antagonist protocols were applied in controlled ovarian hyperstimulated patients. In the agonist protocol, GnRH agonist $0.5 \mathrm{mg} /$ day leuprolide acetate (Lucrin Daily; Abbott, Istanbul, Turkey) was started in the luteal phase of the previous cycle. Moreover, recombinant human follicle stimulated hormone (r-hFSH) follitropin- $\alpha$ (Gonal-F®; Ares Serono, Geneva, Switzerland) or follitropin- $\beta$ (Puregon $\AA$; Organon, Oss, the Netherlands) was added to the protocol on second and third day of the cycle. In the antagonist protocol, r-hFSH was started two or three days of the cycle. Moreover, when the dominant follicle was $14 \mathrm{~mm}$, cetrorelix (Cetrotide ®; Merck Serono, Turkey) was added to the treatment as GnRH antagonist. In both protocols, the development of the follicles was followed by transvaginal ultrasonography (USG) and Estradiol (E2). When the dominant follicle measurement was $\geq 19 \mathrm{~mm}$ or at least three follicles were $\geq 17 \mathrm{~mm}$, gonadotropin administration was discontinued, and ovulation was induced. Ovulation was induced with $250 \mu \mathrm{g}$ of human recombinant hCG (Ovitrelle ${ }^{\circledR}$, Merck
Serono, Turkey) or the GnRH agonist induction which consisted of SC injection of $1 \mathrm{mg}$ leuprolide acetate (Lucrin; Abbott,Istanbul, Turkey) was administered. Oocyte retrieval process was performed with transvaginal USG at between 36th-40th hours after ovulation induction.

Serum and follicular fluid samples were taken into five cc sterile tubes during oocyte retrieval process and centrifuged at $3000 \mathrm{rpm}$ for 10 minutes before being stored. Then, supernatants were separated and stored at $80^{\circ} \mathrm{C}$ until the analysis. Follicular fluid specimens were taken from follicles thought to be only $17 \mathrm{~mm}$ and over.

After all samples were completed, serum and follicular fluid SCF levels were determined by ELISA using the SCF ELISA kit (East Biopharm, Hangzhou, PRC Lot no. 20170324) in the biochemistry laboratory at University of Gazi and the results were expressed as $\mathrm{pg} / \mathrm{mL}$. All of the procedures were performed according to the manufacturers' instructions.

This study was approved by the local Ethics Committee. A written consent form was obtained from all patients and the study was conducted in accordance with the principles of the Declaration of Helsinki.

\section{Statistical analysis}

All statistical analyses were performed using the SPSS for Windows version 11.5 software (SPSS Inc., Chicago, IL, USA). Descriptive statistics were expressed in mean \pm standard deviation (SD) and median (min-max) for numerical variables, and number (percentage) for categorical variables. When to look whether there was a statistically significant difference between the categories of a qualitative variable with two categories in terms of a quantitative variable, Student-t Test was used if the normal distribution assumptions were met; if not, Mann Whitney U test was used. The chi-square and the Fisher exact tests were used to analyze the relationship between two categorical variables. Linear regression analysis was used when it was desired to examine how one or more independent variables affected a dependent variable obtained by the measurement of interest and how the variation on the dependent variable could be explained by means of these independent variables. Significance level was set at $\mathrm{p}=0.05$.

\section{RESULTS}

The demographic characteristics of a total of 132 patients included in this study and the hormonal variables at the beginning of the ICSI cycle are shown comparatively according to the groups, in Table 1. Forty-two (73.7\%) of the PCOS patients included in the study were with primary infertility, $15(26.3 \%)$ with secondary infertility and $69(92.0 \%)$ patients in the control group were with primary infertility, six $(0.8 \%)$ with secondary infertility. In the PCOS group, IVF/ICSI was previously performed 
in three patients, IUI in 30 patients, whereas IVF was previously performed in one patient and IUI in 36 patients in the control group, but pregnancy did not occur.

Table 1: Comparison of age, BMI, pre-cycle hormonal values between PCOS and control groups.

\begin{tabular}{|c|c|c|c|c|c|}
\hline \multirow{3}{*}{ Variables } & \multicolumn{5}{|l|}{ Group } \\
\hline & \multicolumn{2}{|l|}{$\operatorname{PCOS}(\mathrm{N}=57)$} & \multicolumn{3}{|c|}{ Control $(\mathrm{N}=75)$} \\
\hline & Mean \pm SD & Median (Min-Max) & Mean \pm SD & Median (Min-Max) & $\mathrm{p}$ value \\
\hline Age(year) & $29.42 \pm 4.30$ & $29.00(22.00-37.00)$ & $29.76 \pm 4.32$ & $30.00(23.00-38.00)$ & $0.648^{\mathrm{a}}$ \\
\hline BMI $\left(\mathrm{kg} / \mathrm{m}^{2}\right)$ & $25.19 \pm 5.46$ & $23.46(19.13-37.91)$ & $22.41 \pm 2.59$ & $22.00(19.00-27.00)$ & $0.008^{\mathrm{b}}$ \\
\hline FSH (mIU/mL) & $8.07 \pm 2.74$ & $7.95(4.79-16.26)$ & $7.96 \pm 3.03$ & $7.56(3.94-17.30)$ & $0.417^{\mathrm{b}}$ \\
\hline LH (mIU/mL) & $7.22 \pm 4.98$ & $6.40(1.05-23.36)$ & $5.32 \pm 2.61$ & $4.57(1.74-11.33)$ & $0.018^{\mathrm{a}}$ \\
\hline $\mathrm{E} 2(\mathrm{pg} / \mathrm{mL})$ & $44.31 \pm 16.78$ & $41.05(20.00-78.00)$ & $55.11 \pm 21.99$ & $47.56(22.00-107.00)$ & $0.004^{\mathrm{a}}$ \\
\hline TSH (mIU/mL) & $2.01 \pm 0.92$ & $2.00(0.12-3.82)$ & $1.77 \pm 0.64$ & $2.00(0.77-3.00)$ & $0.093^{\mathrm{a}}$ \\
\hline PRL $(\mu \mathrm{g} / \mathrm{L})$ & $17.56 \pm 5.85$ & $18.00(9.22-30.71)$ & $16.10 \pm 7.45$ & $16.57(5.68-35.29)$ & $0.060^{\mathrm{a}}$ \\
\hline $\begin{array}{l}\text { Duration of } \\
\text { infertility (year) }\end{array}$ & $5.94 \pm 2.87$ & $6.25(2.00-14.00)$ & $6.20 \pm 2.30$ & $6.50(2.00-12.00)$ & $0.388^{\mathrm{a}}$ \\
\hline
\end{tabular}

a: Student-t Test; b: Mann Whitney U Test; *p<0.05 statistically significant

Table 2: Comparison of cycle-related parameters between PCOS and control groups.

\begin{tabular}{|c|c|c|c|c|c|}
\hline \multirow{3}{*}{ Variables } & \multicolumn{5}{|l|}{ Group } \\
\hline & \multicolumn{2}{|l|}{$\operatorname{PCOS}(n=57)$} & \multicolumn{3}{|l|}{ Control $(n=75)$} \\
\hline & Mean \pm SD & $\begin{array}{l}\text { Median } \\
\text { (Min-Max) }\end{array}$ & Mean \pm SD & $\begin{array}{l}\text { Median } \\
\text { (Min-Max) }\end{array}$ & $\mathrm{p}$ value \\
\hline Total gonadotropin dose (IU) & $2408.33 \pm 731.42$ & $\begin{array}{l}2250.00 \\
(1475.00-3600.00)\end{array}$ & $2810.29 \pm 1092.73$ & $\begin{array}{l}2700.00 \\
(1000.00-4775.00)\end{array}$ & $0.014^{\mathrm{b}}$ \\
\hline Total cycle duration (day) & $12.27 \pm 1.49$ & $\begin{array}{l}12.00 \\
(10.00-15.00)\end{array}$ & $12.29 \pm 2.76$ & $\begin{array}{l}11.00 \\
(9.00-21.00)\end{array}$ & $0.232^{\mathrm{b}}$ \\
\hline $\begin{array}{l}\text { Total induction duration } \\
\text { (day) }\end{array}$ & $10.27 \pm 1.49$ & $\begin{array}{l}10.00 \\
(8.00-13.00)\end{array}$ & $10.41 \pm 3.16$ & $\begin{array}{l}9.00 \\
(7.00-21.00)\end{array}$ & $0.232^{\mathrm{b}}$ \\
\hline E2 level in hCG Day(pg/mL) & $2807.58 \pm 1139.37$ & $\begin{array}{l}2300.00 \\
(1284.00-4856.00)\end{array}$ & $2910.61 \pm 2760.82$ & $\begin{array}{l}1900.50 \\
(98.00-10958.00)\end{array}$ & $0.049^{b}$ \\
\hline Retrieved oocyte (n) & $15.79 \pm 6.04$ & $\begin{array}{l}15.00 \\
(5.00-28.00)\end{array}$ & $7.96 \pm 3.65$ & $\begin{array}{l}7.00 \\
(1.00-14.00)\end{array}$ & $<0.001^{\mathrm{a}}$ \\
\hline Mature $\left(M_{2}\right)$ oocyte $(n)$ & $10.79 \pm 6.80$ & $\begin{array}{l}11.00 \\
(0.00-24.00)\end{array}$ & $5.52 \pm 3.27$ & $\begin{array}{l}6.00 \\
(0.00-12.00)\end{array}$ & $<0.001^{\mathrm{a}}$ \\
\hline Day 3 grade 1 embryos (n) & $6.93 \pm 4.76$ & $\begin{array}{l}8.00 \\
(0.00-14.00)\end{array}$ & $4.94 \pm 3.23$ & $\begin{array}{l}5.00 \\
(0.00-9.00)\end{array}$ & $0.031^{\mathrm{a}}$ \\
\hline D5 blastocyst (n) & $0.53 \pm 1.60$ & $\begin{array}{l}0.00 \\
(0.00-6.00)\end{array}$ & $0.41 \pm 1.46$ & $\begin{array}{l}0.00 \\
(0.00-6.00)\end{array}$ & $0.774^{\mathrm{b}}$ \\
\hline $\begin{array}{l}\text { Endometrial double wall } \\
\text { thickness (EDWT) (mm) }\end{array}$ & $11.68 \pm 1.65$ & $\begin{array}{l}11.20 \\
(9.50-15.00)\end{array}$ & $10.71 \pm 1.67$ & $\begin{array}{l}10.00 \\
(8.50-14.50)\end{array}$ & $0.061^{\mathrm{a}}$ \\
\hline $\begin{array}{l}\text { Number of embryos } \\
\text { transferred }\end{array}$ & $1.11 \pm 0.32$ & $\begin{array}{l}1.00 \\
(1.00-2.00)\end{array}$ & $1.00 \pm 0.00$ & $\begin{array}{l}1.00 \\
(1.00-2.00)\end{array}$ & $0.104^{b}$ \\
\hline
\end{tabular}

a: Student-t Test b: Mann-Whitney U Test; *p<0.05 statistically significant

Table 2 shows the quantitative parameters of the ICSI cycles comparatively between PCOS and control groups. The agonist protocol was applied to nine patients $(30.0 \%)$ in the PCOS group and 21(70.0\%) patients in the control group, whereas the antagonist protocol was applied to 48 $(47.1 \%)$ in the PCOS group and $54(52.9 \%)$ in the control group.

There was no statistically significant difference between groups in terms of treatment protocol $(\mathrm{p}=0.097)$. For the ovulation induction, $18(24.0 \%)$ patients in the PCOS group and $57(76.0 \%)$ patients in the control group received rec-hCG, whereas an agonist induction was applied to $39(68.4 \%)$ patients in the PCOS group and 18 $(31.6 \%)$ patients in the control group. The agonist induction rate was statistically significant and higher in the PCOS group $(\mathrm{p}=<0.001)$. In Table 3 , the outcomes of ICSI cycles between PCOS and control groups are compared in terms of implantation rates and clinical pregnancy rates.

Serum SCF (sSCF) levels were compared between PCOS and control groups and sSCF level in PCOS group was found to be statistically lower than control group $(\mathrm{p}<0.001)$. 
The median (min-max) values of sSCF level in the PCOS and control groups were found to be 3.61 (0.83-12.00) and 10.17 (1.89-48.85), respectively.

Table 3: Comparison of cycle outcome between PCOS and control groups.

\begin{tabular}{|llll|}
\hline & Group & Control & $\begin{array}{l}\text { p } \\
\text { value }\end{array}$ \\
\hline $\begin{array}{l}\text { Implantation rate / ET } \\
(\%)\end{array}$ & 30.8 & 41.4 & $0.037^{\mathrm{a}}$ \\
\hline $\begin{array}{l}\text { Clinical pregnancy rate/ } \\
\text { ET }(\%)\end{array}$ & 26.3 & 35.8 & $0.041^{\mathrm{a}}$ \\
\hline $\begin{array}{l}\text { Multiple pregnancy, n } \\
(\%)\end{array}$ & $3(5.3)$ & $0(0.0)$ & $0.078^{\mathrm{b}}$ \\
\hline
\end{tabular}

a: Chi-square test $\quad$ b: Fisher's Exact Test; ${ }^{*} p<0.05$ statistically significant

Similarly, follicular fluid SCF (ffSCF) levels were compared between PCOS and control groups and ffSCF level in the PCOS group was found to be statistically lower than control group $(\mathrm{p}<0.001)$. The mean \pm SD values of the ffSCF level in the PCOS and control groups were found to be $10.98 \pm 4.58$ and $30.01 \pm 16.59$, respectively. SCF levels in serum and follicular fluid in PCOS and control groups are shown in Figures 1 and 2.

The relationship between presence/absence of clinical pregnancy and SSCF and ffSCF levels in PCOS and control groups were investigated. As seen in Table 4, sSCF and ffSCF were statistically significantly higher who had clinical pregnancy than those who had no clinical pregnancy in the PCOS group ( $p<0.001$ and $\mathrm{p}=0.027$, respectively). There was no statistically significance between SSCF and ffSCF levels in terms of presence/absence of clinical pregnancy in the control group $(\mathrm{p}=0.732$ and $\mathrm{p}=0.519$ respectively).

At this part of the study the relation between sSCF and ffSCF levels and oocyte maturation were investigated.
The linear regression analysis was performed to examine the effect of SCF levels on serum and follicular fluid on oocyte maturation rate (number of mature oocytes/number of oocytes collected).

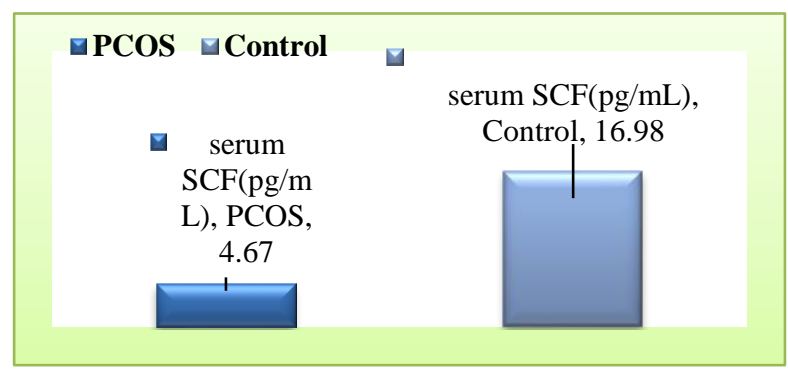

Figure 1. Serum SCF levels in PCOS and control groups.

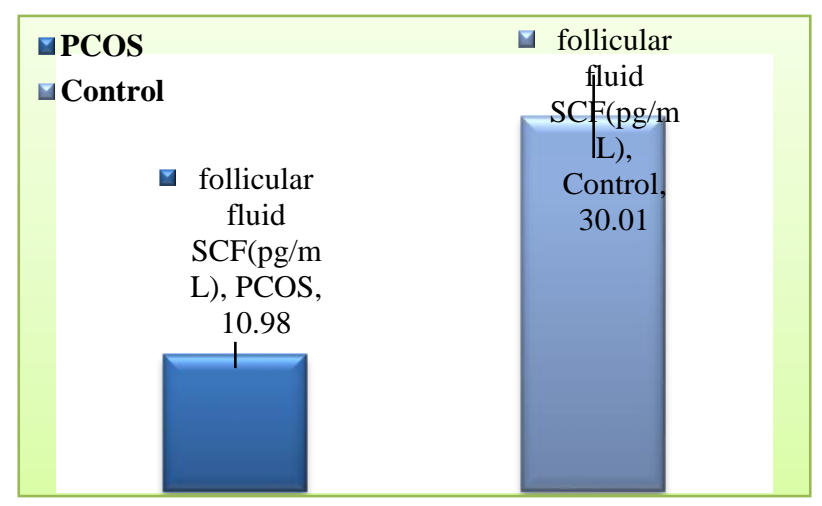

Figure 2. ffSCF levels in PCOS and control group were shown.

This model, in which oocyte maturation ratio was included as a dependent variable and $\mathrm{sSCF}(\mathrm{p}=0.012)$ and ffSCF $(p=0.007)$ as independent variables in the PCOS group, found to be statistically significant $(p=0.017)$. Model of regression was found as Oocyte maturation ratio $=0.392-0.071 \times$ sSCF $+0.054 \mathrm{x}$ ffSCF.

Table 4: Relationships between clinical pregnancy, s SCF and ffSCF in PCOS and control groups.

\begin{tabular}{|c|c|c|c|c|c|}
\hline Groups & Variables & & & Serum SCF & Follicle SCF \\
\hline \multirow{5}{*}{ PCOS } & \multirow{5}{*}{$\begin{array}{l}\text { Clinical } \\
\text { Pregnancy }\end{array}$} & \multirow{2}{*}{ Yes $(n=15)$} & Mean \pm SD & $5.52 \pm 3.20$ & $11.00 \pm 5.11$ \\
\hline & & & Median (Min-Max) & $3.80(1.07-12.00)$ & $10.87(3.17-20.00)$ \\
\hline & & \multirow{3}{*}{ No $(n=42)$} & Mean \pm SD & $2.84 \pm 2.45$ & $8.95 \pm 3.56$ \\
\hline & & & Median (Min-Max) & $2.48(0.83-7.48)$ & $9.61(8.86-18.10)$ \\
\hline & & & $\mathrm{p}$ value & $<0.001 *$ & $0.027 *$ \\
\hline \multirow{5}{*}{ Control } & \multirow{5}{*}{$\begin{array}{l}\text { Clinical } \\
\text { Pregnancy }\end{array}$} & \multirow{2}{*}{ Yes $(n=27)$} & Mean \pm SD & $11.10 \pm 13.19$ & $24.52 \pm 15.25$ \\
\hline & & & Median (Min-Max) & $18.45(4.89-32.17)$ & $30.32(9.88-57.24)$ \\
\hline & & \multirow{3}{*}{ No $(n=48)$} & Mean \pm SD & $19.75 \pm 14.99$ & $32.60 \pm 17.00$ \\
\hline & & & Median (Min-Max) & $17.77(4.11-41.85)$ & $33.06(2.79-45.30)$ \\
\hline & & & $\mathrm{p}$ value & 0.732 & 0.519 \\
\hline
\end{tabular}

$* \mathrm{p}<0.05$ statisticallysignificant 
The R2 value of this model was found to be 0.402 and it can be interpreted that these two independent variables together explain $40.2 \%$ of the change in oocyte maturation rate. SSCF and ffSCF as an independent variable in the control group and the oocyte maturation ratio as a dependent variable were included in the model in which there was no statistically significant difference $(\mathrm{p}=0.634)$.

\section{DISCUSSION}

In this study, serum and follicular fluid SCF levels in PCOS patients were found to be lower than in non-PCOS group. sSCF and ffSCF were statistically significantly higher who had clinical pregnancy than those who had no clinical pregnancy in the PCOS group but there was no statistically significance between SSCF and ffSCF levels in terms of presence/absence of clinical pregnancy in the control group. sSCF and f SCF levels were shown to may be effective on oocyte maturation in the PCOS group. In the non-PCOS group, such a relationship was not found. In the first step of present study, demographic characteristics, hormonal parameters, duration of infertility and ICSI cycle characteristics were compared between PCOS and non-PCOS groups. There was no significant difference between the methods applied when the variables during cycling are examined.

The number of oocytes obtained and the number of mature oocytes as a natural result of this process were higher in the PCOS group than in the non-PCOS group. ${ }^{15}$ Implantation rate and clinical pregnancy rate were lower in the PCOS group as consistent with the literature. ${ }^{16,17}$

In the present study, serum and follicular fluid SCF levels were found to be lower in the PCOS group than in the non-PCOS group. Tan et al reported in their study that serum and follicular fluid SCF levels were lower in PCOS patients than in those without PCOS. In the same study, it was shown that SCF expression in the granulosa cell (GC) decreased due to GC dysfunction in PCOS patients. ${ }^{13}$ Sun et al found that serum and follicular fluid levels of SCF were higher in PCOS patients than nonPCOS group and they concluded that SCF might play a role in the pathogenesis of PCOS. ${ }^{18}$ In the same study, Sun et al reported that they investigated the relationship between IVF outcome and serum and ffSCF levels in IVF cycles of PCOS patients. ${ }^{18}$ As a result of this study, they showed that SCF levels were higher in pregnant of both PCOS and non-PCOS group than in the non-pregnant individuals and they concluded that high levels of SCF in follicular fluid of IVF patients may be beneficial on oocyte maturation, embryo development and blastocyst implantation. ${ }^{18}$

In support of this interpretation, Tan et al that increases of SCF levels in granulosa cells and follicular fluid increased oocyte maturation, fertilization and embryo quality. ${ }^{14}$ Smikle et al reported about 20 years ago that the increase in ffSCF levels of PCOS group increased the
IVF success and made the following comment in 1998: "Therefore, stem cell factor may play a role in human follicular and oocyte development, and increasing infrafollicular stem cell factor concentrations may improve pregnancy rates."19 In a similar manner, we also found higher serum and follicular fluid SCF levels in the presence of clinical pregnancy group in PCOS patients but we found no statistically significant difference nonPCOS group.

Inspired by literature finding suggests that SCF may be associated with oocyte maturation, and in the regression, analysis associated therewith, we found that both serum SCF and ffSCF levels would be effective on oocyte maturation, this relationship was observed only in PCOS patients, but not in the non PCOS group. In the study conducted by Gizzo et al, they reported that the increase in serum SCF levels in poor responder patients was positively correlated with the rate of mature oocytes. ${ }^{20}$

First in the literature, this study suggests that SCF levels are low in serum and follicular fluid in patients with PCOS and that the increase in SCF levels is associated with an increase in oocyte maturation and clinical pregnancy rates in PCOS. As a limitation, in present study, follicular fluid was taken from the follicles thought to be only mature, nevertheless how these mechanism work in the immature follicles is a candidate for future researches.

\section{CONCLUSION}

Serum and follicular fluid SCF levels are decreased in PCOS, however increased levels of SCF in patients with PCOS are associated with an increased oocyte maturation and clinical pregnancy rates. It is possible that treatment strategies targeting SCF to improve oocyte maturation and pregnancy rates in PCOS can be developed in the future.

This study suggests that SCF levels are low in serum and follicular fluid in patients with PCOS and that the increase in SCF levels is associated with an increase in oocyte maturation and clinical pregnancy rates in PCOS. In present study, follicular fluid was taken from the follicles thought to be only mature, while in the immature follicles it is a candidate for research into how these mechanisms work. It is possible that treatment strategies targeting SCF to improve oocyte maturation and pregnancy rates in PCOS can be developed in the future.

\section{Funding: No funding sources \\ Conflict of interest: None declared \\ Ethical approval: The study was approved by the Institutional Ethics Committee}

\section{REFERENCES}

1. Trikudanathan S. Polycystic ovarian syndrome. Med Clin North America. 2014;99(1): 221-35. 
2. Eshre TR, ASRM-Sponsored PCOS Consensus Workshop Group. Revised 2003 consensus on diagnostic criteria and long-term health risks related to polycystic ovary syndrome. Fertil Steril. 2004;81(1), 19-25.

3. Eshre TT, ASRM-Sponsored PCOS Consensus Workshop Group. Consensus on infertility treatment related to polycystic ovary syndrome. Fertil Steril. 2008;89(3), 505-22.

4. Kalem, MN, Kalem Z, Gürgan T. Problems and Complications During the Treatment of Infertility in Women with Polycystic Ovary Syndrome. Gynecol Obstet Reprod Med, 2016;22(2):113-24.

5. De Leo V, Musacchio MC, Cappelli V, Massaro MG, Morgante G, Petraglia F. Genetic, hormonal and metabolic aspects of PCOS: an update. Reprod Biol Endocrinol. 2016;14(1):38.

6. Reddy P, Shen L, Ren C, Boman K, Lundin E, Ottander U, et al. Activation of Akt (PKB) and suppression of FKHRL1 in mouse and rat oocytes by stem cell factor during follicular activation and development. Develop Biol. 2005;281(2):160-70.

7. Liu K. Stem cell factor (SCF)-kit mediated phosphatidylinositol 3 (PI3) kinase signaling during mammalian oocyte growth and early follicular development. Front Biosci. 2006;11:126-35.

8. Raucci F, Di Fiore MM. Localization of c-kit and stem cell factor (SCF) in ovarian follicular epithelium of a lizard, Podarcis s. sicula. Acta Histochemica. 2011;113(6):647-55.

9. Zhang X, Zhang H, Gao Q, Ji S, Bing L, Hao J. Sohlh2 inhibits the apoptosis of mouse primordial follicle oocytes via C-kit/PI3K/Akt/Foxo3a signalling pathway. Reprod Biomed Online. 2015;30(5):514-21.

10. Hu R, Wang FM, Yu L, Luo Y, Wu X, Li J, Zhang $\mathrm{XM}$, Oehninger S, Bocca S. Antimüllerian hormone regulates stem cell factor expression in human granulosa cells. Fertil Steril. 2014;102(6):1742-50.

11. Jin $X$, Han CS, Yu FQ, Wei P, Hu ZY, Liu YX. Anti-apoptotic action of stem cell factor on oocytes in primordial follicles and its signal transduction. Mol Reprod Develop: Incorporating Gamete Res. 2005;70(1):82-90.

12. Tan J, Wen XY, Su Q, Huang ZH, He JX, Xin CL, Wu QF, Zou Y. Reduced expression of SCF in serum and follicle from patients with polycystic ovary syndrome. Eur Rev Med Pharmacol Sci. 2016;20(24):5049-57.

13. Tan J, Zou Y, Wu XW, Tian LF, Su Q, et al. Increased SCF in follicular fluid and granulosa cells positively correlates with oocyte maturation, fertilization, and embryo quality in humans. Reprod Sci. 2017;24(11):1544-50.

14. Qiao J, Feng HL. Extra-and intra-ovarian factors in polycystic ovary syndrome: impact on oocyte maturation and embryo developmental competence. Hum Reprod Update. 2010;17(1):17-33.

15. Ozeren M, Dinc H, Ekmen U, Senekayli C, Aydemir V. Umbilical and middle cerebral artery Doppler indices in patients with preeclampsia. Eur J Obstet Gynecol Reprod Biol. 1999;82:11-6.

16. Child TJ, Abdul-Jalil AK, Gulekli B, Tan SL. In vitro maturation and fertilization of oocytes from unstimulated normal ovaries, polycystic ovaries, and women with polycystic ovary syndrome. Fertil Steril. 2001;76(5):936-42.

17. Mikkelsen AL, Lindenberg S. Benefit of FSH priming of women with PCOS to the in vitro maturation procedure and the outcome: a randomized prospective study. Reproduction. 2001;122(4):58792.

18. Sun LJ, Sun HX, Qian QR, Yue LF, Wang XL, Guan YC, et al. Correlationship between SCF, TGF- $\beta 1$ level in serum and follicular fluid with PCOS pathogenesis and IVF outcome In PCOS patients [J]. Chinese J Birth Health Heredity. 2012;10:053.

19. Smikle CB, Dandekar PV, Schriock ED, Givens CR. Elevated ovarian follicular fluid stem cell factor concentrations are associated with improved pregnancy rates in in-vitro fertilization cycles. Fertil Steril. 1998;69(1):70-2.

20. Gizzo S, Quaranta M, Andrisani A, Bordin L, Vitagliano A, Esposito F, et al. Serum stem cell factor assay in elderly poor responder patients undergoing IVF: a new biomarker to customize follicle aspiration cycle by cycle. Reprod Sci. 2016;23(1):61-8.

Cite this article as: Kalem Z, Kalem MN, Seval M, Bakirarar B, Simsir C, Yilmaz C, et al. Serum and follicular fluid concentration of stem cell factor in PCOS. Int J Reprod Contracept Obstet Gynecol 2018;7:3467-72. 